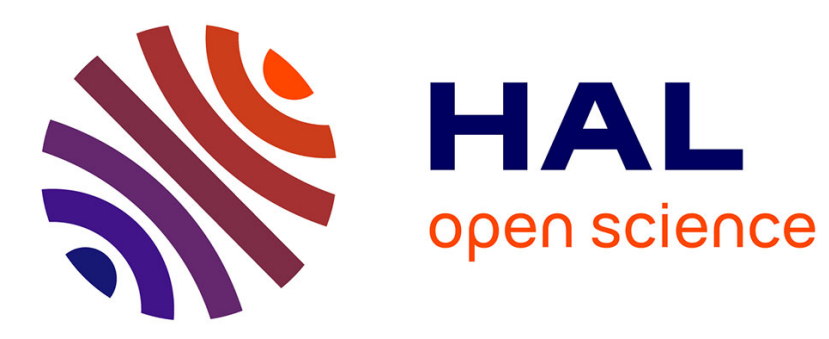

\title{
Masculin/féminin. Le genre des technologies de l'information
}

\author{
Chantal Morley
}

\section{To cite this version:}

Chantal Morley. Masculin/féminin. Le genre des technologies de l'information. Revue Française de Gestion, 2004, 30 (148), pp.67 - 86. 10.3166/rfg.148.67-86 . hal-02550935

\section{HAL Id: hal-02550935 https://hal.science/hal-02550935}

Submitted on 22 Apr 2020

HAL is a multi-disciplinary open access archive for the deposit and dissemination of scientific research documents, whether they are published or not. The documents may come from teaching and research institutions in France or abroad, or from public or private research centers.
L'archive ouverte pluridisciplinaire HAL, est destinée au dépôt et à la diffusion de documents scientifiques de niveau recherche, publiés ou non, émanant des établissements d'enseignement et de recherche français ou étrangers, des laboratoires publics ou privés. 


\title{
Revue française de gestion, vol.30/148, janv.-fév. 2004. Masculin / féminin : le genre des technologies de l'information
}

\author{
Chantal Morley, Maître de conférences à l'INT (Groupe des Ecoles de \\ Télécommunication)
}

\section{Introduction}

La fille de Lord Byron, Ada Lovelace(1815-1852), ne se doutait pas que son prénom serait un jour attribué à un langage informatique. Passionnée de mathématiques, elle conceptualisa, un siècle avant les premiers calculateurs électroniques, les bases d'une machine programmable.

En revanche, les femmes qui ont brillamment pénétré le domaine nouveau de l'informatique au milieu du XXe siècle, pensaient sans doute que ce secteur d'activités s'ouvrirait largement aux femmes. Citons notamment les «ENIAC girls », mathématiciennes recrutées dans les années 40 pour programmer le premier ordinateur. Ou bien Grace M.Hopper ${ }^{1}$, qui a conçu en 1949 le premier langage de haut niveau ${ }^{2}$ et en a écrit le premier compilateur. Ou encore sœur Mary K.Keller, première femme à obtenir en 1965 un doctorat en informatique, qui a participé au développement du langage Basic ${ }^{3}$ [Gürer, 1995 ; Tijdens, 1997].

Or, en ce début de XXIe siècle, les femmes sont largement minoritaires dans les métiers de l'informatique. Il y a quelques années, d'après un rapport européen, leur nombre ne dépassait pas 30\% [Social Europe, 1993]. Aujourd'hui encore, cette sous-représentation, commune à tous les pays [Baroudi,1995; Tijdens,1997; ETAN, 1999], conduit à s'interroger [Panteli,1997 ; Igbaria,1997]. On observe notamment une stagnation, voire une diminution des étudiantes en informatique [Klawe, 1995 ; Oechtering, 1995 ; Schinzel,1997 ; Rasmussen, 1997 ; Greenhill, 1997]. Les entreprises de la nouvelle économie n'ont pas fondamentalement modifié la donne [Godat, 2000] ; en France, on compte 3\% de créatrices de jeunes pousses pour $30 \%$ dans l'ensemble des autres secteurs de l'économie [Grésy, 2000]. La tendance actuelle dans les technologies de l'information ne semble pas devoir renverser la situation [Catalyst, 2001].

Ce phénomène pose trois problèmes.

1. L'explosion des activités autour des technologies de l'information, conjuguée à la pénurie de personnels compétents dans les métiers de l'informatique, devrait conduire à utiliser au mieux toutes les ressources potentielles [Panteli,1999].

2. Le retrait des femmes est peut-être à mettre en relation avec d'éventuelles pratiques discriminatoires, alors qu'il y avait un consensus pour penser que les activités modernes échappaient à un héritage marqué par la ségrégation hommes/femmes [Truman, 1994].

\footnotetext{
${ }^{1}$ On lui doit l'origine du mot « bug » appliqué à l'informatique, pour avoir écrit dans le journal de bord en 1945, après une panne d'un relai de l'ordinateur causée par la présence d'une mite : «First actual case of bug being found », qui a conduit son équipe à parler de «debugging »l'ordinateur. [Gürer, 1995].

${ }^{2}$ Ce langage servira de modèle pour le langage Cobol, quelques années plus tard.

${ }^{3}$ Pour elle le centre de calcul de l'Université de Dartmouth a dérogé à la règle «men only ».
} 
3. Il semble que la diversité des employés (âge, sexe, race) soit un facteur de performance pour l'entreprise, en particulier dans le secteur des technologie de l'information : se priver de femmes pourrait ainsi réduire le potentiel de l'entreprise [Panteli,1999].

Pour tenter d'expliquer ce phénomène, nous allons l'aborder par une question plus large: quelle relation y a-t-il entre les technologies, en particulier les technologies de l'information, et le masculin / féminin. Les technologies sont-elles neutres ou sont-elles par essence masculines?

\section{L'article est ainsi organisé.}

Nous allons d'abord préciser la différence entre les termes sexe et genre.

Puis, nous présenterons les trois grandes façons d'aborder la relation technologie et masculin / féminin : le point de vue essentialiste, le point de vue socio-culturel et le point de vue interactioniste. Ces trois points de vue correspondent à des courants de recherches, mais on peut aussi les considérer comme trois regards, dont aucun n'épuise totalement la compréhension de la relation technologie - genre. Sociologiquement, les chercheurs sont de deux origines. Certains sont des chercheurs en informatique qui se préoccupent de la participation limitée des femmes dans leur domaine et s'intéressent particulièrement à la formation et aux initiatives pour en accroître le nombre. Les Anglo-saxons appellent ce courant «women in technology ». L'autre courant, nommé "gendering of technology », est plutôt composé de chercheurs en sciences sociales, qui utilisent une approche sociologique et/ou philosophique [Adam, 1995 ; Wübker, 1997]. Un pont entre ces deux courants a été jeté par l'IFIP ${ }^{4}$ en 1994, par la tenue d'un colloque réunissant les deux populations de chercheurs.

Nous replacerons enfin la relation entre technologie et genre dans une perspective anthropologique.

\section{Les termes « sexe » et « genre »}

Les deux termes «sexe » et «genre » sont parfois employés de façon synonyme. Leur définition a fait l'objet de débats récents en psychologie [Gentile, 1993 ; Unger, 1993 ; Deaux, 1993].

On considérera que le terme «sexe»s'applique aux différences biologiques entre hommes et femmes.

Le terme «genre »s'applique à la différence entre masculin et féminin. Il renvoie à des comportements psychologiques et sociaux, comme les stéréotypes de genre et les rôles de genre. Le stéréotype de genre est un ensemble de caractéristiques psychologiques et comportementales qu'un groupe social attribue généralement à un homme (stéréotype de genre masculin) ou à une femme (stéréotype de genre féminin).

Les rôles de genre sont les catégories de rôles que l'on considère, dans une société donnée, comme convenant à un homme (rôles de genre masculin) ou à une femme (rôles de genre féminin). Quand on veut parler des rôles effectivement tenus par des hommes ou des femmes, on parlera de rôles de sexe [Lueptow, 1995].

À partir des années 30, un courant de recherche a considéré qu'en réalité tout individu, quel que soit son sexe, possède des caractéristiques masculines et des caractéristiques féminines. Différentes échelles ont été mises au point pour mesurer le degré de masculinité et le degré de féminité d'une personne. Par exemple, l'échelle de Bem [Bem, 1974] distingue trois catégories d'items, masculins, féminins et neutres. Ces items représentent des comportements jugés socialement souhaitables dans la société américaine, soit pour des

\footnotetext{
${ }^{4}$ International Federation for Information Processing.
} 
hommes, soit pour des femmes, ou bien indifféremment pour les deux. Un extrait en est donné dans la figure 1.

Ainsi, un individu de sexe masculin peut-il être fortement féminin et une personne de sexe féminin peut-elle être fortement masculine. Dans de tels cas, la personne se trouvera en décalage, non pas physiologiquement, mais socialement. En effet, il est communément admis qu'il doit y avoir une adéquation entre le genre et le sexe. Comme le définit R.K.Unger, «le terme de genre peut être utilisé pour désigner les composantes non physiologiques du sexe qui sont actuellement perçues comme appropriées aux individus de sexe masculin ou aux individus de sexe féminin » (Female and Male : Psychological Perspective, cité et traduit par [Le Maner-Idrissi, 1997] p.17).

\begin{tabular}{|l|l|l|}
\hline \multicolumn{1}{|c|}{ Items masculins } & \multicolumn{1}{c|}{ Items féminins } & \multicolumn{1}{c|}{ Items neutres } \\
\hline Se comporte en leader & Affectueux (se) & S'adapte facilement \\
\hline Agressif (ve) & Gai (e) & Vaniteux (se) \\
\hline Ambitieux (se) & A une âme d'enfant & Consciencieux (se) \\
\hline Analytique & Compatissant (e) & Conventionnel (le) \\
\hline Sûr (e)de soi dans ses affirmations & Ne fait pas usage d'un langage cru & Amical (e) \\
\hline Athlétique & $\begin{array}{l}\text { Empressé (e) à alléger les peines } \\
\text { d'autrui }\end{array}$ & Heureux (se) \\
\hline Défend ses idées & Accessible à la flatterie & Serviable \\
\hline Dominateur (trice) & Doux (ce) & Inefficace \\
\hline Energique & Crédule & Jaloux (se) \\
\hline Plein de force & Aime les enfants & Aimable \\
\hline Apte au commandement & Loyal (e) & Lunatique \\
\hline Indépendant (e) & Sensible aux besoins des autres & Digne de confiance \\
\hline Individualiste & Timide & Réservé (e) \\
\hline Prend facilement des décisions & Parle d'une voix douce & Sincère \\
\hline Confiance en soi & Va vers les autres & Grave \\
\hline Se suffit à soi-même & Tendre & Plein (e) de tact \\
\hline Forte personnalité & Compréhensif (ve) & Comédien(ne) \\
\hline $\begin{array}{l}\text { Résolu (e) dans ses prises de } \\
\text { position }\end{array}$ & Chaleureux (se) & Franc (che) \\
\hline Cherche à prendre des risques & Accomodant (e) & Imprévisible \\
\hline Masculin & Féminin & Fantaisiste \\
\hline
\end{tabular}

Figure 1 : Échelle de Bem

Nous allons donc examiner selon trois points de vue la relation entre le genre et les technologies de l'information, pour tenter de comprendre pourquoi les personnes de sexe féminin sont en grande partie éloignées des métiers des technologies de l'information.

\section{Premier point de vue : Le point de vue essentialiste}

Ceux qui adoptent ce point de vue considèrent que la nature des femmes s'oppose à la nature masculine des technologies. Explicitement ou non, ils admettent une forme de déterminisme biologique. Les plus radicaux, parfois appelés les éco-féministes [Grint et Woolgar, 1995], considèrent que les femmes, par leur rôle dans la reproduction, sont en communion avec la nature. Inversement, les hommes cherchent à dominer la nature. Les technologies que nous connaissons en sont une expression, elles sont donc étrangères aux femmes. Ceci est particulièrement vrai, mais pas exclusivement, des technologies tournées vers la guerre. Selon ce point de vue, la question du genre ne se pose pas, car sexe et genre se confondent.

Ce point de vue n'est pas propre aux féministes radicales, mais imprègne en réalité beaucoup d'expressions de pensée, rattachées ou opposées à une idéologie féministe. La 
différence de nature entre hommes et femmes, avec les caractéristiques psychologiques et comportementales spécifiques à chaque sexe, est une idée qui a imprégné une majorité de cultures. Elle a été notamment développée par Aristote qui a profondément marqué la culture occidentale. Nous allons en donner quelques illustrations.

De nombreux discours sur les hommes et les femmes dans l'entreprise évoquent une nature particulière. Ainsi, dans un numéro des Cahiers de l'ENSPTT consacré aux femmes dirigeantes en entreprise [Les Cahiers, 1998], on peut en trouver différents exemples: «L'entreprise, dans une large majorité, a compris l'intérêt qu'elle avait à jouer la mixité, et donc la complémentarité homme/femme, la conjugaison du rationnel et de l'intuitif $»^{5}$. Ou bien : «Les hommes sont très bons pour tout ce qui touche le 'cerveau gauche' et le domaine rationnel... et les femmes, le 'cerveau droit' et l'intuition. À chacun son domaine.» ${ }^{6}$. Ou encore : «Les qualités narcissiques féminines sont essentielles, à la condition que les femmes en entreprise soient 'femmes' et n'essaient pas de battre les hommes sur leur terrain.» ${ }^{7}$.

Le point de vue essentialiste se retrouve également dans les recherches de L.Irigaray sur les différences d'expression linguistique entre hommes et femmes dans plusieurs langues européennes [Irigaray, 1990], car l'auteur présente ces différences dans l'absolu, sans prendre en compte d'autre paramètres que le sexe comme par exemple l'âge, le milieu social ou la formation.

Certaines recherches sur les stéréotypes de genre incluent un point de vue essentialiste. Ainsi au États-Unis, des chercheurs ayant trouvé que, malgré la profonde évolution des rôles et attitudes des femmes dans la société, les perceptions des caractéristiques homme/femme ont peu évolué en vingt ans et que les sexes se conforment aux stéréotypes de genre, concluent : «Il nous semble que les explications socio-psychologiques doivent prendre en compte les influences génétiques qui se font jour si clairement dans les travaux contemporains ${ }^{8} \gg$ [Lueptow, 1995].

Plus centrées sur l'informatique, citons les recherches qui s'appuient sur le modèle d'acceptation technologique $(\mathrm{TAM})^{9}$ en y rajoutant la variable «sexe » [Venkatesh, 2000 ; Gefen, 1997]. Ce modèle postule que l'acceptation et l'utilisation d'une nouvelle technologie est déterminée par deux croyances clés : l'utilité perçue et la facilité d'usage perçue (Fig.2). Ce qui peut se traduire par les deux questions suivantes : "La nouvelle technologie va-t-elle améliorer ma performance au travail ? (utilité perçue) et «L'utilisation de la nouvelle technologie ne nécessite-t-elle qu'un faible effort de ma part ?» (facilité d'usage perçue).

\footnotetext{
${ }^{5}$ Interview de G. Le Gallenec, Directrice Générale d'EOS Conseil, p.32.

${ }^{6}$ Interview de S. Pelissier, Conseiller du Président à La Poste, p.48.

${ }^{7}$ Interview de D.Sibony, psychanalyste, p.120.

${ }^{8}$ «It seems to us that social psychological explanations must include the genetically fixed influences that are so clearly emerging in contemporary work».

${ }^{9}$ TAM : Technology Acceptance Model.
} 


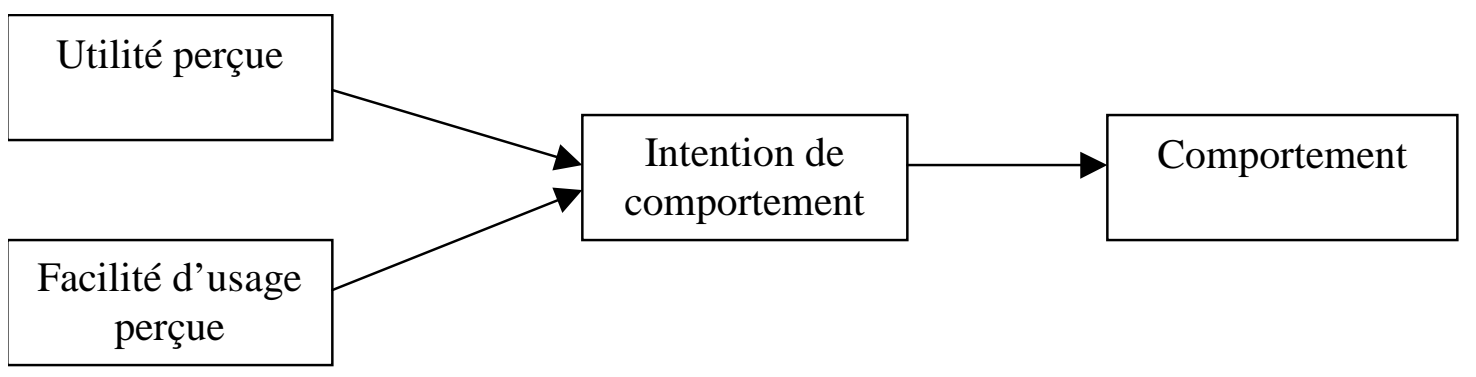

Figure 2 : Modèle d'acceptation technologique (TAM)

Les auteurs qui étendent le modèle en introduisant la variable homme/femme font l'hypothèse que le sexe influe sur les deux croyances clés. Ainsi, [Gefen, 1997] a trouvé que les hommes et les femmes ont une utilisation semblable, mais une perception différente du courrier électronique. [Venkatesh, 2000], étudiant l'introduction d'une nouvelle application de gestion d'un référentiel, a trouvé que les hommes sont davantage déterminés par l'utilité perçue, alors que les femmes sont déterminées à parts égales par les deux croyances clés.

Certes, les auteurs parlent non pas de «nature», mais de «culture» et s'appuient notamment sur les travaux de G.Hofstede qui a mesuré les différences culturelles dans le monde du travail entre 40 pays à l'aide de quatre variables, dont le «degré de masculinité », défini comme les dispositions envers les attitudes et comportements masculins [Hofstede, 1980]. Les auteurs font l'hypothèse que si les dispositions vis-à-vis des stéréotypes de genre permettent de définir une culture nationale (a priori partagée par les hommes et les femmes de ce pays), alors ces mêmes stéréotypes de genre doivent influencer l'attitude des hommes et des femmes vis-à-vis des technologies de l'information. D'une culture nationale, on passe ainsi de façon plus fine à une culture de sexe (Fig. 3), peut-être transnationale.

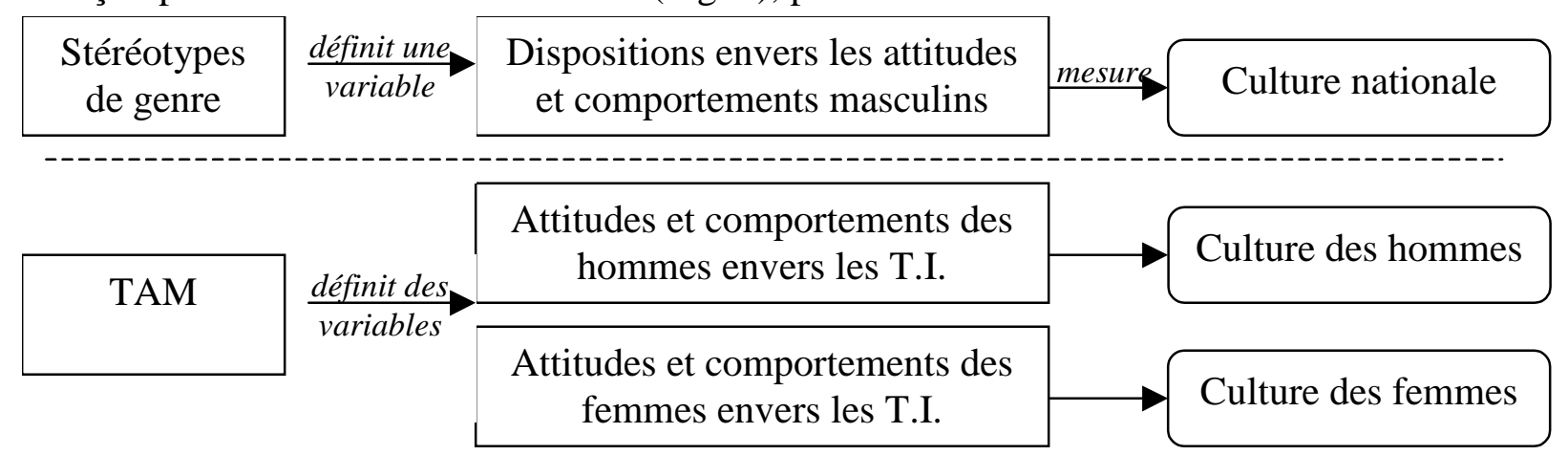

Figure 3 : Passage de la notion de culture nationale à la notion de culture sexuée

Ceci n'est pas, soulignons-le, ce qui ressort de l'étude de G.Hofstede, qui montre au contraire que le «degré de masculinité » est corrélé, non pas au sexe, mais à la position hiérarchique [Hofstede, 1987 p.143] et à l'âge [Hofstede, 1987 p.191].

Les auteurs qui ont étudié les différences hommes/femmes dans l'acceptation technologique, en tirent des recommandations pratiques, notamment pour les managers et les formateurs, les incitant à avoir des approches et des discours différenciés pour les hommes et pour les femmes lors de l'implantation de nouvelles technologies de l'information dans les entreprises. "Managers who are aware of this difference can better align with users's culture, an alignment that could improve IS implementation success » [Gefen, 1997, p.398]. Le sexe comme élément de culture est ici très proche de la nature, puisque l'on considère qu'il y a correspondance intangible entre sexe et comportement de genre. «While men still represent a majority of the work force, the number of women at all levels of the organizational hierarchy continues to rise. Therefore, technology acceptance theories and models that overlook gender 
as an important factor may overestimate the influence of productivity-oriented factors while simultaneously underestimating the importance of ease of use perceptions and social influences $\gg$ [Venkatesh, 2000].

Le facteur sexe semble avoir une influence en soi et de façon atemporelle. On peut donc considérer que ces deux recherches sont influencées par le point de vue essentialiste.

En ce qui concerne le rapport aux technologies, les tenants les plus radicaux de ce point de vue appellent à se tenir à distance de la technologie: "the more radical view is that technology is so essentially masculine that no change could really demasculinize it » [Grundy, 1996, p.139]. D'autres, plus modérés, préfèrent inventer des applications tournées vers les femmes, en particulier par une introduction accrue de l'informatique dans la sphère domestique, par exemple des bases de données culinaires ou médicales, en local ou via internet [Grundy, 1996, p.36 à 42]. Cependant, plutôt qu'une approche différente de la technologie, les femmes semblent s'intéresser à des champs différents: "There is some evidence that women try to develop or design technology differently from men, but this is the result of a tendency to choose different problem fields rather than solving problems/producing designs that are different » [Hapnes, 1995 p.176].

\section{Deuxième point de vue : le point de vue socio-culturel}

Ce point de vue repose sur deux assertions. D'une part, la technologie est neutre ; d'autre part, hommes et femmes partagent la même humanité et la même rationalité. L'environnement social et les processus de socialisation conduisent les hommes et les femmes à tenir des rôles différents. Les modèles de comportement présentés par la société expliquent les freins à la formation et à l'accès aux technologies.

De nombreuses études ont été faites autour des stéréotypes de rôles, en particulier par des chercheurs en informatique, que ce soit dans la formation ou dans les métiers de l'informatique.

\subsection{Les stéréotypes dans la formation informatique}

De façon générale, il apparaît que les filles issues d'établissements d'enseignement supérieur non mixtes réussissent mieux dans la préparation aux carrières connotées masculines - mathématiques, sciences, commerce - que celles qui sont dans des établissements mixtes [Klawe, 1995 ; Durand-Delvigne, 1998]. Divers facteurs explicatifs ont été avancés.

Les enseignants, hommes et femmes, ont souvent des comportements différents envers les filles et envers les garçons, plus attentifs et plus stimulants envers ces derniers [Grundy, 1996 p.72 ; Klawe, 1995].

À performance égale, les filles ont moins d'assurance que les garçons [Klawe, 1995 ; Lundeberg, 1994], particulièrement en ce qui concerne l'informatique considérée comme le territoire des garçons [Grundy, 1996 p.73]. «Les filles ont tendance à sous-estimer leurs potentialités dans des domaines traditionnellement connotés masculins lorsqu'elles sont en présence de garçons » [Durand-Delvigne, 1998 p.84]. De façon plus générale, c'est dans les situation de mixité que les filles développent une image d'elles-mêmes proche des stéréotypes féminins » [Durand-Delvigne, 1998].

Ce sentiment est fondé ou renforcé par l'inégalité d'accès à l'ordinateur familial, les garçons apparaissant comme des utilisateurs prioritaires sinon exclusifs [Oechtering, 1995 ; Schinzel, 1997], ce qui peut s'expliquer par le faits que la majorité des jeux semblent s'adresser à des garçons [Grundy, 1996 p.14]. 


\subsection{Les stéréotypes dans les métiers de l'informatique}

\section{Les inégalités}

Les inégalités entre hommes et femmes dans les métiers de l'informatique sont une réalité, aussi bien en Europe qu'en Amérique [Panteli, 1999 ; Igbaria, 1997 ; Tijdens,1997 ; Klawe, 1995 ].

On explique parfois les différences de salaire et de niveau hiérarchique par les écarts entre la valeur du capital humain représenté par les hommes et celle du capital humain que représentent les femmes. Selon cette perspective, les femmes parce qu'elles ont un taux de turn-over plus élevé, davantage d'interruptions de carrière, moins d'expérience, un niveau de formation plus bas, moins de mobilité, moins de formation continue (à cause des responsabilités familiales), ne font pas progresser autant que les hommes la valeur de leur capital humain [Truman, 1994 ; Baroudi, 1995]. Certaines recherches montrent cependant qu'à niveau professionnel, âge, niveau de formation et expérience professionnelle égaux, les femmes reçoivent un salaire plus faible, notamment aux Etats-Unis [Truman, 1994] ou en Grande-Bretagne [Igbaria, 1997].

Une autre explication, avancée dès les années 60, des inégalités constatées entre hommes et femmes, est l'attitude au travail, c'est-à-dire un engagement moindre des femmes qui n'accordent pas la même valeur au travail que les hommes. Cependant, d'après des recherches plus récentes, il semble qu'actuellement, à âge, niveau de formation, type de poste et revenu équivalents, hommes et femmes réagissent de façon similaire [Lefkowitz, 1994]. Plus précisément dans le domaine informatique, les aspirations en termes de responsabilité, d'intérêt du travail et de challenge proposé, apparaissent semblables chez les hommes et les femmes [Panteli, 1999].

Ainsi, d'après les recherches menées au cours des années 90, rien n'indique que la situation d'égalité hommes/femmes soit meilleure dans l'informatique que dans les autres secteurs. En effet, les femmes sont systématiquement sous-représentées aux rangs de direction, même à âge, expérience et niveau de formation égaux; elles sont moins payées que les hommes pour un même niveau hiérarchique et l'écart s'accroît quand on monte dans la hiérarchie [Hemenway, 1995].

\section{Les évolutions de carrière}

Les recherches sur la carrière des femmes en informatique font souvent référence au «plafond de verre », mécanisme subtil et quasi invisible qui bloque l'avancement des femmes [Meyerson, 2000 ; Hemenway, 1995]. On observe le même phénomène dans les milieux de l'enseignement supérieur et de la recherche en informatique [Klawe, 1995 ; Grundy, 1996 p.75], comme plus largement dans les domaines scientifiques [Boukhobza, 2000].

Les formes subtiles de discrimination qui bloquent l'avancement de femmes sont souvent accidentelles, non intentionnelles et non reconnues. [Hemenway, 1995] a analysé de façon fine comment les stéréotypes de genre, en vigueur dans la société, freinent l'évolution professionnelle des femmes, notamment en informatique. Nous allons en indiquer les principaux points.

D'abord, la notion de la réussite professionnelle est davantage associée à un homme qu'à une femme. C'est particulièrement vrai dans les disciplines scientifiques ou d'ingénierie. Cela peut conduire à des situations perdant-perdant pour les femmes. Si elles adoptent un style de travail traditionnellement féminin — recherche de consensus et de travail de groupe - on les juge moins compétentes et moins assurées. Si, au contraire, elles optent pour un comportement plus agressif, elles déplaisent, car elles apparaissent masculines. Une étude d'une association américaine a montré que le type de personnalité apte à réussir dans 
beaucoup de sociétés d'ingénierie est beaucoup plus agressif que ce que la plupart des hommes dans les entreprises tolèrent pour une femme [Catalyst, 1992].

Ensuite, l'attitude conversationelle peut être influencée par les stéréotypes : faut-il marquer des points (domination) ou rechercher une coopération (conciliation) ? Certaines femmes, parce qu'elles se tiennent en dehors du jeu verbal, peuvent perdre une compétition sans même se douter qu'il y avait compétition. Une analyse du contenu des échanges sur un forum électronique dans un contexte de formation continue à distance a fait apparaître des comportements différents, largement marqués par les stéréotypes de genre [Blum K.D.,1999]. Parfois, la domination conversationnelle se double d'un biais de surdité : seules les idées émises par des hommes sont entendues.

Certains comportements de managers relèvent également des stéréotypes de genre qui freinent l'avancement. Ils évitent de confier aux femmes des tâches comportant un défi à relever, supposant implicitement qu'elles ne sont pas ambitieuses et qu'il n'est donc pas important de leur donner des opportunités pour progresser. De leur côté, les femmes adoptent plus facilement un comportement attendant la récompense en silence, alors que les hommes réclament agressivement [Hemenway, 1995].

Les stéréotypes sociaux ont aussi un impact sur l'évaluation des performances. La psychologie sociale explique ainsi le phénomène [Terborg, 1975]. Il est communément admis que les récompenses doivent être proportionnelles aux contributions. Cependant, le jugement sur les réalisations est entaché d'incertitude, il faut donc faire des interprétations ou des projections. C'est alors que peuvent intervenir les préjugés sur les femmes et les hommes, conduisant à dévaloriser les contributions des femmes. Ceci a été confirmé par des études montrant que lorsqu'il n'y a pas d'incertitude, alors les biais ne jouent pas. Un exemple de biais est cité dans le domaine de l'enseignement supérieur français par [Boukhobza, 2000], évoquant l'âge comme un frein au recrutement et à l'évolution de carrière des femmes : «Pour les femmes entre 30 et 40 ans, on invoque la question de la maternité. Après 40 ans, elles sont trop âgées pour être enseignantes-chercheures car soupçonnées d'être inactives dans la recherche. Elles sont à ce titre peu considérées. À l'inverse, les hommes au fil de l'âge semblent gagner en sagesse et en savoir scientifique. Cela suppose que les femmes ne capitalisent pas de savoir scientifique !». Un autre exemple est donné par une recherche menée en Suède dans le domaine médical, qui a montré que pour devenir professeur une femme doit avoir produit 2,6 fois plus de publications qu'un homme [ETAN, 1999 p.46].

L'observation de la répartition existante des rôles peut conduire à forger des images mentales qui renforcent les stéréotypes. Ainsi, un rôle professionnel - directeur informatique, chef de projet, professeur... - peut être inconsciemment associé à des caractéristiques personnelles qui n'ont pas a priori de rapport avec les tâches et responsabilités du poste. Dans la recherche de candidats potentiels, on va spontanément faire appel des personnes possédant ces caractéristiques. Les femmes sont elles-mêmes biaisées par ces images mentales, qui peuvent les empêcher de chercher à occuper une position plus élevée, considérant plus ou moins consciemment que cela ne leur convient pas [Hemenway, 1995]. L'absence de modèle de rôle empêche le processus de socialisation anticipatoire, c'està-dire l'adoption par une personne qui aspire à rejoindre un groupe des comportements de ce groupe (habillement, manières...). Ceci peut être interprété comme un manque de compétence ou de qualification de la part des femmes [Hemenway, 1995].

\subsection{Les différences de stratégies de carrière}

Le problème du temps de travail (dépassements d'horaires, réunions tardives) et de son rapport au temps privé ont été souvent évoqué pour ses «effets discriminants sur la carrière » des femmes [Belloni, 1998 ; Boukhobza, 2000]. Ces questions qui relèvent de la division 
sociale et familiale du travail domestique sont capitales. Cependant, leur prise en compte n'épuise pas l'explication des carrières différenciées. Les stratégies d'avancement de carrière comportent une dimension tacite, d'autant plus importante que le contexte est informel. Nous allons en donner trois éléments qui ont été étudiés dans le contexte informatique: l'appartenance à un réseau social, la valorisation différentielle des travaux et la pratique du parrainage (mentoring).

\section{Le réseau social}

M.Tierney a analysé l'évolution de carrière des personnels dans la filiale irlandaise d'un constructeur de matériel informatique, plus précisément dans un service offrant du support et du développement logiciel pour les clients. Le contexte de travail y est moderne, informel, décontracté, peu hiérarchisé, sans barrières - par exemple l'encadrement n'a pas de bureau séparé pour favoriser une communication directe. Cependant, à compétences égales, tous ne progressent pas de la même façon : l'élément décisif est l'existence et l'appartenance à un réseau informel de jeunes programmeurs, brillants, ayant des centres d'intérêt communs (football), sortant régulièrement ensemble, et entretenant de bonnes relations avec l'encadrement. Ce réseau offre une source d'information précieuse sur les postes disponibles, un soutien pour les nouveaux arrivés dans l'accomplissement des tâches et une aide pour discerner les rôles qu'il est préférable d'occuper pour faire carrière. Tous les hommes ne sont pas admis dans ce réseau informel, mais aucune femme n'en fait partie. [Tierney, 1995].

F.Grundy a observé pendant un an le service informatique d'une direction régionale de la santé en Grande-Bretagne, en participant aux travaux de programmation. Dans un contexte de travail décloisonné, avec du personnel jeune, les hommes occupent l'espace verbal (choix des sujets de discussion, volume sonore, vocabulaire) s'assurant ainsi une position dominante [Grundy, 1996 p.49-51]. L'accumulation de ces comportements collectifs peut conduire les femmes à une auto-dévalorisation et leur demander un surcroît d'énergie pour trouver leur place dans un environnement perçu comme hostile [Hemenway, 1995].

L'importance jouée par les relations sociales est à rapprocher d'une étude menée aux États-Unis sur l'acceptation du travail à distance [Bélanger, 1999]. Les hommes y apparaissent nettement défavorables, invoquant le besoin de partager des informations avec des collègues et le besoin d'avoir des relations sociales. Les femmes se montrent plus favorables, pour des raisons liées au travail domestique qui leur incombe, au détriment de leur stratégie de carrière.

\section{La valorisation différentielle des travaux}

Certains travaux informatiques sont moins valorisés (par exemple, élaborer la documentation d'une application, assurer la formation des utilisateurs, faire la maintenance d'une application, faire la reprise de bases de données pour les adapter à un nouveau système) ou moins visibles (par exemple, adapter les sorties d'une progiciel pour répondre au mieux aux besoins des utilisateurs), donc moins pertinents dans l'évolution de carrière. Ces travaux semblent être confiés de préférence à des femmes [Grundy, 1996 p.58-62], certains allant jusqu'à parler d'un phénomène de "ghetto » dans le développement informatique [Panteli, 1997].

La notion de travail invisible [Balka, 1997] renvoie à ces activités qui remettent les choses sur rail face à des imprévus : on le qualifie de travail d'articulation. Comme ce type de travail ne figure pas dans les modèles rationnels, on ne le reconnaît pas dans les projets de développement de système d'information. Son absence peut toutefois conduire à l'échec du système, notamment pour les systèmes supportant le travail coopératif [Robertson, 1997]. Comme la plus grande part du travail d'articulation est accompli par des femmes, la non- 
reconnaissance de ce type de travail renforce l'invisibilité de la contribution du travail des femmes.

La stratégie d'évitement des tâches peu valorisées ou leur acceptation rejoint l'observation de N.Boukhobza, H. Delavault et C. Hermann au sujet des freins à l'évolution

de carrière des femmes dans l'enseignement supérieur en France: «Les femmes s'investissent beaucoup dans l'enseignement. C'est un élément important pour elles plus que pour les hommes. Malheureusement, il n'est pas valorisé dans le dossier de l'évolution de carrière »; de plus, en dehors de l'enseignement et la recherche, les femmes sont plutôt à des postes de gestionnaires, alors que les hommes sont à des postes plus stratégiques, plus visibles : «les femmes sont dans les bureaux, les hommes dans les instances de décision ». Elles se demandent si les femmes «ne reproduisent pas des fonctionnements qui s'opèrent dans leur espace privé » [Boukhobza, 2000].

\section{Le parrainage}

Un mentor peut être défini guide bienveillant dans l'institution qui fait éviter les embûches et montre les meilleurs chemins et stratégies de succès [Klawe, 1995]. Les anglosaxons appellent mentoring la relation entre un cadre confirmé (le mentor) et un cadre d'un niveau inférieur (le protégé), qui vise à aider le protégé à comprendre les rouages de l'entreprise pour monter dans la hiérarchie. Cette relation n'a pas pour finalité la transmission de connaissances techniques [Pfleeger, 1995]. Elle joue un rôle essentiel pour l'avancement dans un certain nombre d'entreprises.

Cependant, la psychologie sociale a montré que dans les interactions sociales et professionnelles, les contraires ne s'attirent pas. Cette tendance au repli identitaire affecte les femmes, car cela conduit les mentors potentiels à choisir de jeunes collègues qui leur ressemblent [Hemenway, 1995]. Comme les femmes sont moins nombreuses aux postes de cadres confirmés et que leur position est parfois moins influente que ne le laisserait penser leur titre - phénomène d'alibi ou tokenism [Truman, 1994] — les femmes sont beaucoup moins souvent que les hommes aidées dans leur progression professionnelle.

$\mathrm{Au}$ niveau académique, les femmes trouvent rarement des mentors qui les présentent à des agences susceptibles de financer des recherches ; celles qui tentent de le faire seules sont considérées arrivistes, ou se sentent mal à l'aise d'avoir un comportement aussi agressif [Klawe, 1995].

Pour conclure sur ce deuxième point de vue, les stéréotypes de genre ont une influence importante dans la formation, les métiers et les carrières professionnelles, notamment dans les technologies de l'information. Comme l'exprime C.Baudelot, "ethnologues et sociologues ont montré depuis plus d'un demi-siècle que, par leurs formes de socialisation distinctes, les hommes et les femmes sont porteurs depuis leur prime enfance de valeurs différentes.(...) Idéologiquement érigés en qualité naturelles, ces deux ordres de valeur ont été exploités productivement dans la division du travail entre les sexes (...). Le grand intérêt des travaux de sciences sociales réalisés sur cette question a précisément consisté à mettre en évidence le caractère arbitraire et socialement construit de ce partage » [Baudelot, 1998 p.281-282]. La réponse se trouve dans des campagnes de communication et des actions d'incitation pour lever les barrières posées par les stéréotypes de rôles masculins/féminins [ETAN, 1999]. Si elles veulent avoir une relation à la technologie égale à celle des hommes, les femmes doivent répondre de façon positive à ces appels et changer leur propre vision des rôle de genre. Certaines entreprises ont favorisé le développement interne d'un réseau de femmes [Diwanji, 1995]. Certains pensent qu'Internet est également un moyen d'informer et soutenir professionnellement les femmes [Grundy, 1997 p.113-147]. 


\section{Troisième point de vue : Le point de vue interactioniste}

Selon ce point de vue, il y a une interaction entre l'image de la technologie et l'affirmation de soi-même comme masculin ou féminin [Gill, 1995]. D'un côté, la technologie apparaît comme porteuse d'attributs masculins. D'un autre côté, l'attitude envers la technologie permet d'affirmer son identité masculine ou féminine.

\subsection{L'image de la technologie face au genre}

L'idée que l'on se fait de la masculinité comporte de nombreux attributs que l'on associe à la technique : la force, la sûreté, la détermination, la rationalité. Toute technologie augmente la force physique ou intellectuelle, les capacités, les pouvoirs de la personne qui la maîtrise. L'informatique augmente la capacité de mémorisation et de recherche d'information, la puissance de calcul, le pouvoir de communication.

Par un glissement d'image, la force que donne la technologie la dote de caractéristiques masculines. Qui n'a jamais pensé qu'un ordinateur était sûr de lui, dominateur, peu sensible aux besoins des autres, résolu dans ses prises de positions, faisant même parfois usage d'un langage brutal [Grundy, 1996] ${ }^{10}$ ? Or, ce sont autant d'attributs marquant le masculin dans la grille de Bem (Fig.1).

Ainsi, ce qui est perçu comme technologique est associé à des attributs masculins. La participation des femmes dans la conception ou l'utilisation des technologies ne modifie pas cette association, comme le souligne F.Murray: «What is perceived to be technological is perceived to be masculine. That is, if masculinity fails to control or looses control of technological practices, those practices then lose their status as technological practices » [Murray, 1992 p.67]. Dans le domaine des technologies domestiques, on peut citer le cas du four à micro-onde, qui a d'abord été commercialisé en ciblant les hommes célibataires avec une image de nouveauté technologique — «produit brun» — et qui, en se banalisant, a changé de cible et d'image — «produit blanc» - pour devenir un simple accessoire de cuisine [Grundy, 1996 p.30]. La programmation a d'abord été perçue comme un travail administratif, donc largement ouvert aux femmes : quand on a réalisé, dans les années 70, que la vraie puissance de l'informatique dépendait du logiciel, les femmes sont devenues plus rares [Grundy, 1996 p.48].

\subsection{L'affirmation du genre par la technologie}

Être attiré par les technologies, s'en rendre familier, les maîtriser, sont des attitudes et comportements qui sont considérés comme des caractéristiques du genre masculin. Inversement, le manque d'attirance et de compétence technique est perçu comme constitutif de l'identité féminine. Utiliser ou rejeter les technologies est donc une façon d'affirmer son genre, «doing gender », selon l'expression de West et Zimmerman [West, 1987], signifiant par là que le genre relève peut-être davantage des comportements — du faire — que de la nature — de l'être [Gill, 1995 ; Gisler, 1997].

Certains chercheurs considèrent que l'incompétence technique des femmes est une construction sociale [Hirata, 1998]. Cette incompétence peut toutefois être revendiquée pour affirmer son genre, de la même façon que certains hommes se déclarent incompétents dans la maîtrise de certains objets techniques domestiques, fer à repasser ou machine à coudre.

\footnotetext{
${ }^{10}$ Par exemple, en anglais, « access violation, integrity degradation, chaining records, to abort a program, to kill a job ».
} 
En ce qui concerne plus précisément les technologies de l'information, le glissement de l'informatique comme application des mathématiques vers les sciences de l'ingénierie est considéré par certains [Schinzel, 1997] comme une des raisons majeures en Allemagne de la diminution des étudiantes en informatique. Dans la même direction, C.Marry, considérant le nombre de filles dans les filières scientifiques et d'ingénieurs plus important en France qu'en Allemagne, a émis l'hypothèse que le système français des écoles d'ingénieurs est plus généraliste que notamment les Fachhochschulen allemandes (écoles techniques supérieures), ce «qui occulte pour partie leur connotation 'masculine' : le 'technique' est encore moins affaire de femmes que le 'scientifique' » [Marry, 1998 p.78]. Les chercheuses en informatique tendent à qualifier leurs compétences de non techniques, ce qui n'est pas toujours apparent, sans d'ailleurs expliciter le notion de compétence technique [Erb, 1997].

Le phénomène «hacker » est un exemple du rôle de la technologie dans l'affirmation de l'identité, qui a été étudié par plusieurs chercheurs [Håpnes, 1995].

Les hackers sont des jeunes hommes, possédés par une passion pour l'informatique. On les trouve le plus souvent dans un environnement universitaire. Leur mode de travail est marqué par l'indépendance : ils rejettent toute méthode de programmation et sont fortement opposés aux langages structurés qui bloquent leur créativité en leur préférant le $\mathrm{C}$ ou le langage machine; ils ne jurent que par les stations de travail et dédaignent les PC ou les Macintosh, les premiers trop orientés vers une utilisation uniforme, les seconds jugés snobs, féminins et pleins d'obstacles à leur créativité. Leur but majeur est de maîtriser les machines et d'en être le vainqueur: devant une idée ou un problème, leur grand plaisir sera d'en programmer une solution informatique. Ce sont de grands amateurs de jeux se pratiquant via le réseau ${ }^{11}$ et au cours desquels chaque joueur adopte et se construit une identité fictive. Le travail nocturne est souvent apprécié. Leur relation à l'ordinateur est donc à la fois recherche de contrôle et développement de leur créativité, à des fins utilitaires comme pour des jeux.

En ce qui concerne leurs relations internes, l'esprit de compétition est très important. Les concours d'endurance permettent de distinguer celui qui a réussi à programmer le plus longtemps possible sans s'arrêter. Mais la coopération est aussi un élément important de la culture pour tout ce qui touche à la programmation : les hackers détestent les secrets et les protections des ordinateurs et logiciels.

Dans leurs relations avec l'extérieur, ils se perçoivent très tôt comme ayant des intérêts différents de ceux des filles, comme en témoigne l'un d'eux : "The first thing you learn as a computer boy is : Never talk to girls about computers! They get this kind of desperate expression. I believe they think it's too complicated, they cannot stand to listen to it. All of us belonging to this hacker community have learnt that. » [Håpnes, 1995 p.186-187].

On observe ainsi qu'à travers leurs relations entre eux, avec les autres groupes et avec l'ordinateur, ces jeunes gens construisent leur masculinité.

Les groupes de hackers sont minoritaires parmi les étudiants en informatique. De plus, ils ont un fonctionnement interne non autoritaire et non individualiste, donc non complètement marqué «masculin». Cependant, par leur visibilité et par leur composition faite quasi exclusivement d'hommes, ils contribuent à l'association entre informatique et masculin, ce qui augmente le retrait des filles [Schizel,1997] qu'elles justifient par une absence d'intérêt pour le domaine [Rasmussen, 1997]. De façon moins excessive que dans le phénomène des « hackers », il semble que l'ordinateur familial soit également un moyen chez les jeunes garçons de constituer un réseau masculin leur permettant de s'affirmer et maintenant les filles en dehors de cette sphère [Foreman,1997].

\footnotetext{
${ }^{11}$ Par exemple, MUD (Multi-User Dungeon).
} 
Ainsi, la technologie, et notamment la technologie informatique, n'est pas seulement faite d'artefacts, mais aussi de connaissances nécessaires, de pratiques, de relations sociales, d'images : elle est donc porteuse d'une certaine culture qui se présente ou qui se construit comme étrangère pour les femmes. Les éléments de pouvoir n'en sont pas absents. Pratiquer le genre à travers la technologie, c'est symboliquement renoncer à du pouvoir et à de la maîtrise sur les artefacts. "By 'doing gender', men do domination and women do give in. In this way, doing gender reinforces and legitimates hierarchical arrangements. » [Gisler, 1997 p.219].

Certains ont proposé de faire évoluer l'image masculine de l'informatique, en y introduisant de la «féminité » par une redéfinition des concepts fondamentaux, tels que système d'information et communication, ainsi qu'en abordant certains points souvent occultés tels que l'objectivité/subjectivité de l'information, les effets de l'abstraction, la prise en compte des structure réelles de communication, les relations entre le formel et l'informel, la place du désordre et des conflits... [Crutzen, 1997].

\section{La relation technologie et genre dans une perspective anthropologique}

Quel que soit le point de vue adopté, la relation entre sexe et genre est une question centrale pour comprendre comment se maintient et se reproduit le lien entre masculinité et technologie. L'anthropologie sociale peut nous apporter des éclairages.

Il semble en effet que tout au cours de l'histoire de l'humanité, le genre se révèle comme une construction sociale, basée sur la répartition sexuelle des tâches et sur des énoncés symboliques - mythes, discours idéologiques... - qui visent à expliquer l'ordre social [Héritier-Augé, 1996, p.21].

A contrario, certaines sociétés offrent des exemples d'une dissociation entre sexe et genre. Ainsi, chez les Inuits (Eskimos), le genre d'un nouveau-né dépend non pas de son sexe biologique, mais de celui de l'ancêtre qui a pénétré son âme, qu'on appelle «âme-nom ». Jusqu'à la puberté, l'enfant peut donc être élevé sur un mode opposé à son sexe, et cela touche environ 30\% d'entre eux. À la puberté, l'adolescent doit adopter, souvent douloureusement, comportement et habillement de son sexe, tout en conservant l'identité de son âme-nom [Héritier-Augé, 1996, p.202].

Le genre n'est donc pas de l'ordre du naturel. S.Bem, dans ses recherches sur l'androgynie psychologique, a cherché à démontrer deux hypothèses. D'abord, un individu peut être à la fois masculin et féminin, selon les situations. C'est pourquoi elle a proposé non pas une échelle bipolaire, mais deux échelles non exclusives. Ensuite, un individu fortement typé (masculin ou féminin) a un comportement plus rigide et moins adaptatif qu'un individu plus androgyne qui peut choisir son genre de comportement selon les cas [Bem, 1974].

L'existence de l'androgynie va dans le sens de certaines recherches en technologie de l'information. Ainsi, les différences de style de communication dans la communication électronique ne semblent pas prouvées. Deux chercheurs ont ainsi trouvé que le style typiquement masculin (compétition) ou typiquement féminin (coopératif) ne correspond qu'à une minorité d'hommes ou de femmes : dans la plupart des cas, la distribution et le dosage masculin / féminin se font indépendamment du sexe des personnes [Pohl, 1997].

Reste posée la question de l'inégalité masculin / féminin.

F.Héritier-Augé considère que masculin et féminin sont deux catégories fondamentales de l'humanité. Elle fait l'hypothèse que la différence biologique entre les sexes a représenté la base de toute pensée de la différence et a forgé les raisonnements en couples d'éléments 
opposés : masculin / féminin, chaud / froid, sec / humide, clair / obscur, haut / bas, positif / négatif ${ }^{12} \ldots$

Universellement et de tous temps, semble-t-il, on trouve une différence de valeur entre les deux catégories fondamentales. C'est ce que F.Héritier-Augé appelle la valence différentielle des sexes, «un rapport conceptuel orienté, sinon toujours hiérarchique, entre le masculin et le féminin, traduisible en termes de poids, de temporalité (antérieur / postérieur) et valeur» [Héritier-Augé, 1996 p.24]. C'est cette valence qui conduit à penser les technologies comme masculines, si largement immatérielles soient-elles comme l'informatique.

Comment expliquer ce déséquilibre dans le couple masculin / féminin ? F.HéritierAugé, qui a étudié le rôle symbolique du corps humain, l'explique par une différence biologique qu'elle exprime en opposant le «sang des femmes » et le «sang des guerriers » [Héritier-Augé, 1996, p.205]. Les femmes, entre puberté et ménopause, voient leur sang couler, alors que les hommes ne perdent le leur qu'en acceptant la chasse, le combat, la guerre. «Il se pourrait que ce soit dans cette inégalité-là : maîtrisable versus non maîtrisable, voulu versus subi, que se trouve la matrice de la valence différentielle des sexes, qui serait donc elle aussi inscrite dans le corps, dans le fonctionnement physiologique, ou qui procéderait, plus exactement, de l'observation de ce fonctionnement physiologique » [Héritier-Augé, 1996, p.26].

Or, la notion de maîtrise est liée à la définition gendrée de la technologie. De façon générale, les femmes ont été écartées de la maîtrise des techniques. Certains récits ethnographiques font apparaître des exceptions qui toutes concernent des femmes en dehors des périodes de fécondité. Par exemple, chez les Indiens Ojibwa d'Amérique du Nord, les veuves qui ne se remarient pas sont autorisées à acquérir les techniques masculines permettant leur autonomie (chasse, pêche, fabrication de canoë) [Héritier-Augé, 1996, p.246].

La valence différentielle des sexes doit être rapprochée du fait que, dans les entreprises, les valeurs qualifiées de féminines sont louées dans le discours, mais rarement récompensées dans les faits. "Characteristics that are admired such as open communication and collaborative approach are not necessarly promoted $»$ [Panteli, 1999 p.179].

Par ailleurs, en entreprise, la masculinité d'un homme n'est jamais mise en question : le refus de la technologie sera attribué à des goûts personnels et non à son appartenance à un sexe. Comme le remarque C.Marry, «en entreprise, lorsque l'on parle d'une femme, il est toujours question de sa féminité, alors que lorsqu'il s'agit d'un homme, la question de sa masculinité ne se pose pas. Tout ce qui est de l'ordre de l'homme est neutre sexuellement $»^{13}$. Ce que F.Héritier-Augé exprime de façon plus générale en disant qu' "il n'existe pas de catégories dans le modèle du masculin ${ }^{14}$, alors qu'il existe des figures types de femmes (mère, sainte, courtisane...).

\section{Conclusion}

Quelles sont finalement les causes de la distance entre les femmes et les technologies, notamment les technologies de l'information ? La question est complexe et nous avons essayé d'en démêler les différents fils. Par nature ou par culture, les rôles de femmes apparaissent en retrait dans les relations de domination et d'agressivité, et par conséquent en retrait de la maîtrise des technologies qui pourraient assurer ou augmenter leur pouvoir. Les catégories du masculin et du féminin sont universellement positionnées dans un rapport déséquilibré. La

\footnotetext{
${ }^{12}$ On retrouve, par exemple, ces catégories dans les principes yin et yang du taoïsme chinois.

${ }^{13}$ Interview de C.Marry, in [Les Cahiers, 1998 p.97].

${ }^{14}$ Interview de F.Héritier-Augé, in [Les Cahiers, 1998 p.97].
} 
technologie a alimenté ce déséquilibre, en devenant un moyen de construction d'identité, ainsi qu'un instrument de constitution de groupes ou métiers fermés.

Les technologies de l'information, bien que porteuses aussi bien de finalités de domination que de finalités de communication, participent à l'inégalité sociale entre hommes et femmes via l'éducation, la formation, mais aussi les images mentales, les stéréotypes sociaux, les rôles, mais également les métiers, les stratégies de carrière, la distribution du pouvoir. Concevoir des technologies différentes, aborder autrement la définition et l'utilisation des technologies, favoriser une appropriation féminine des technologies, développer un rapport non gendré aux technologies ou changer sa perception ouvrent peutêtre la voie d'une évolution.

\section{Références}

Adam A. (1995), «Women and Computing in the UK », Communications of the ACM, Vol. 58, $\mathrm{N}^{\circ} 1$, p.43.

Balka E. (1997), « Sometimes Texts Speak Louder Than Users : Locating Invisible Work Through Textual Analysis », in Grundy A. et al., 1997, p.163-175.

Baroudi J.J. et Igbaria M. (1995), « An Examination of Gender Effects on Career Success of Information Systems Employees », Journal of Management Information Systems, Winter, Vol.11, `3, P.181201.

Baudelot C. (1998), « Rien n’est joué», in Maruani M. (1998), p.277-283.

Bélanger F. (1999), «Workers'propensy to telecommute : An empirical study », Information \&Management, $\mathrm{N}^{\circ} 35, \mathrm{p} .139-153$.

Belloni C., Boulin J.Y. et Junter-Loiseau A.(1998), « Du temps de travail au temps de la ville», in Maruani M. (1998), p.169-182.

Bem S.L. (1974), «The measurement of psychological androgyny », Journal of Consulting and Clinical Psychology, 42, 2, p.155-162.

Blum K.D. (1999), « Gender Differences in Asynchronous Learning in Higher Education : Learning Styles, Participation Barriers and Communication Patterns », JALN, Vol.3, Issue 1, http://www.aln.org/alnweb/journal/Vol3_issue1/blum.htm .

Boukhobza N., Delavault H. et Hermann C. (2000), «Les enseignants-chercheurs à l'Université », rapport à la Directrice de l'enseignement Supérieur au ministère de l'Education Nationale, mars, http://www.education.gouv.fr/syst/egalite.

Catalyst (1992), «Women in Engineering : An Untapped resource », http://www.catalystwomen.org.

Catalyst (2001), « Women in Information Technology », Infobrief, http://www.catalystwomen.org.

Crutzen C. (1997), « Giving Room to Feminity in Informatics Education », in Grundy A. et al., 1997, p.177-187.

Deaux K. (1993), « Commentary : Sorry, wrong number - A reply to Gentile’s call », Psychological Science, 4, p.125-126.

Diwanji P. (1995), «Corporate Supported Women's Group », Communications of the ACM, Vol. 58, $\mathrm{N}^{\circ} 1, \mathrm{p} .36$.

Durand-Delvigne A. et Duru-Bellat M. (1998), « mixité scolaire et construction du genre», in Maruani M. (1998), p.83-92.

Erb U. (1997), «Exploring the Excluded. A Feminist Approach to Opening New Perspectives in Computer Science », in Grundy A. et al., 1997, p.201-207.

ETAN (1999), European Technology Assesment Network, « Science policies in the European Union : Promoting excellence through mainstreaming gender equality », working paper, http://www.cordis.lu/etan.

Foreman D., Grundy F. et Lees S. (1997), « Sex, Age and Desirability of Computers », in Grundy A. et al., 1997, p.99-110.

Gefen D. et Straub D.W. (1997), « Gender Differences in the Perception and Use of E-Mail : an Extension to the Technology Acceptance Model », MIS Quarterly, décembre, p.389-400.

Gentile D.A. (1993), «Just what are sex and gender, anyway? A call for a new terminological standard », Psychological Science, 4, p.120-122.

Gill R. et Grint K. (1995), « The Gender-Technology Relation. Contemporary theory and research » in Grint K. et Gill R., 1995, p.1-28.

Gisler P. (1997), « Does gender Still Matter? Bodily Functions in Cyberspace : a Feminist Approach », in Grundy A. et al., 1997, p.219-220. 
Godat S. (2000), « Les start-up entrouvent leurs portes aux femmes », Le Monde Interactif, 18 octobre, p.X.

Greenhill A., von Hellens L., Nielsen S. et Pringle R. (1997), « Australian Women in IT Education : Multiple Meanings and Multiculturalism », in Grundy A. et al., 1997, p.387-397.

Grésy B. (2000), Intervention à l'ouverture du Colloque « Sciences et technologies : pourquoi les filles ? », CNAM, 26 octobre.

Grint K. et Gill R. (Eds.)(1995), The Gender-Technology Relation. Contemporary theory and research. Taylor\&Francis, Londres/Washington D.C..

Grint K. et Woolgar S. (1995), « On Some Failures of Nerve in Constructivist and Feminist Analyses » in Grint K. et Gill R., 1995, p.48-75.

Grundy F. (1996), Women and Computers, Intellect Books.

Grundy A.F., Köhler D., Oechtering V. et Petersen U. (Eds.) (1997), Women, work and Computerization : Spinning a Web from Past to Future, Proceedings of the $6^{\text {th }}$ International IFIP-Conference, Bonn, Germany, 24-27 mai, Springer.

Gürer D.W. (1995), « Pioneering Women in Computer Science », Communications of the ACM, Vol. 58, $\mathrm{N}^{\circ} 1, \mathrm{p} .45-54$.

Håpnes T. et Sørenson K.H. (1995), « Competition and Collaboration in Male Shaping of Computing : A Study of a Norwegian Hacker Culture », in Grint K. et Gill R., 1995, p.174-191.

Hemenway K. (1995), « Human Nature and the Glass Ceiling in Industry », Communications of the ACM, Vol. 58, $\mathrm{N}^{\circ} 1$, p.55-62.

Héritier-Augé F. (1996), Masculin-Féminin : la pensée de la différence, Odile Jacob.

Hirata H. et Kergoat D. (1998), « La division sexuelle du travail revisitée », in Maruani M. (1998), p.93104.

Hofstede G. (1980), Culture's Consequences : International Differences in Work Related Values, Sage, Londres. Traduction et adaptation française : Bollinger D. et Hofstede G. (1987), Les Différences culturelles dans le management : comment chaque pays gère-t-il ses hommes? Editions d'organisation, Paris.

Igbaria M., Parasuraman S. et GreenHaus J.H. (1997), « Status report on women and men in the IT workplace », Information Systems Management, Summer, p.44-53.

Irigaray L. (1990), Sexes et genres à travers les langues. Elements de communication sexuée, Grasset, 1990.

Klawe M. et Leveson N. (1995), «Women in Computing : Where Are We Now ?», Communications of the ACM, Vol. 58, N¹, p.29-35.

Lefkowitz J. (1994), «Sex-related differences in job attitudes and dispositional variables : now you see them... », Academy of Management Journal, Vol.37, N², p.323-349.

Le Maner-Idrissi G. (1997), L’identité sexuée, Les Topos, Dunod.

Les Cahiers (1998), Pouvoir au Féminin, avril, ENSPTT.

Lueptow L.B. et Garovich L. (1995), « The Persistence of Gender Stereotypes in the Face of Changing Sex Roles : Evidence Contrary to the Sociocultural Model », Ethology and Sociobiology, Vol.16, $\mathrm{N}^{\circ} 6$, p.509-530.

Lundeberg M.A., Fox P.W. et Puncochar J. (1994), « Highly Confident but Wrong : Gender Differences and Similarities in Confidence Judgments », Journal of Educational Psychology, Vol. 86, $\mathrm{N}^{\circ} 1$, p.114-121.

Marry C. (1998), «La comparaison France-Allemagne à l'épreuve des faits », in Maruani M. (1998), p.71-82.

Maruani M. (1998), Les nouvelles frontières de l'inégalité. Hommes et femmes sur le marché du travail, La Découverte.

Meyerson D.E. et Fletcher J.K. (2000), « Pulvériser l'invisible plafond de la disparité », L'Expansion Management Review, Décembre, p.6 à 16.

Murray F. (1992), « A Separate Reality : Science, Technology and Masculinity », in E.Green, J.Owen et D.Pain (eds), Gendered by design ?, Taylor\&Francis, Londres/Washington D.C..

Oechtering V. et Behnke R. (1995), «Situations and Advancement Measures in Germany », Communications of the ACM, Vol. 58, $\mathrm{N}^{\circ} 1, \mathrm{p} .75-82$.

Panteli A., Ramsay H. et Beirne M. (1997), «Engendered Software Development : Ghettoization and Agency », in Grundy A. et al., 1997, p.305-315.

Panteli A., Stack J., Atkinson M. et Ramsay H. (1999), « The status of women in the UK IT industry : an emprical study », European Journal of Information Systems, N8, p.170-182.

Pfleeger S.L. et Mertz N. (1995), «Executive Mentoring : What make it Work?», Communications of the ACM, Vol. 58, N¹, p.63-73. 
Pohl M. et Michaelson G. (1997), «I don't think that's an interesting dialogue. - Computer-Mediated Communication and Gender », in Grundy A. et al., 1997, p.87-97.

Rasmussen B. (1997), « Girls and Computer Science : It's not me. I'm not Interested in Sitting Behind a Machine all day », in Grundy A. et al., 1997, p.379-386.

Robertson T. (1997), « And it's a Generalisation. But no it's not. Women, Communicative Work and the Discourses of Technology Design » in Grundy A. et al., 1997, p.263-275.

Schinzel B. (1997), «Why has Female Participation in German Informatics Decreased ? » in Grundy A. et al., 1997, p.365-378.

Social Europe (1993), «Occupational Segregation of Women and Men », in the European Community Supplement, 3/93, p.70-76.

Terborg J.R. et Ilgen D.R. (1975), « A theoratical approach to sex-discrimination in traditionally masculine occupations », Organizational Behavior and Human Performance, 13, p.352-376.

Tierney M. (1995), « Negotiating a Software Career : Informal Work Practices and ' The Lads' in a Software Installation », in Grint K. et Gill R., 1995, p.192-209.

Tijdens K. G. (1997), « Gender Segregation in IT Occupations », in Grundy A. et al., 1997, p.449-462.

Truman G.E. et Baroudi J.J. (1994), « Gender Differences in the Information Systems Managerial Ranks : An Assessment of Potential Discriminatory Practices », juin, P.129-143.

Unger R.K. et Crawford M. (1993), «Commentary : Sex and gender. The troubled relationship between terms and concepts », Psychological Science, 4, p.123-124.

Venkatesh V. et Morris M.G. (2000), «Why Don't Men Ever Stop to Ask for Directions ? Gender, Social Influence, and their role in Technology Acceptance and Usage Behavior », MIS Quarterly, Vol.24, $\mathrm{N}^{\circ} 1, \mathrm{p} .115-139$.

West C. et Zimmerman D. (1987), « Doing gender », Gender and Society, vol.1, p.125-151.

Wübker S. et Sigrist B. (1997), « Technology Structuration : A Research Paradigma for a Gender Perspective on Technology ? », in Grundy A. et al., 1997, p.317-323. 